\title{
Intermittent regimens for tuberculosis treatment: Back to the Future?
}

\author{
Jan-Willem C. Alffenaar ${ }^{1,2,3}$, Simon Tiberi ${ }^{4}$, Daniela M. Cirillo ${ }^{5}{ }^{5}$ and \\ Giovanni Battista Migliori $\mathbb{1}^{6,7}$
}

Affiliations: ${ }^{1}$ Faculty of Medicine and Health, School of Pharmacy, University of Sydney, Sydney, Australia. ${ }^{2}$ Westmead Hospital, Sydney, Australia. ${ }^{3}$ Marie Bashir Institute for Infectious Diseases and Biosecurity, University of Sydney, Sydney, Australia. ${ }^{4}$ Dept of Infection, Royal London Hospital, Barts Health NHS Trust, London, UK. ${ }^{5}$ Emerging Bacterial Pathogens Unit, IRCCS San Raffaele Scientific Institute, Milan, Italy. ${ }^{6}$ Servizio di Epidemiologia Clinica delle Malattie Respiratorie, Istituti Clinici Scientifici Maugeri IRCCS, Tradate, Italy. 'Blizard Institute, Queen Mary University of London, London, UK.

Correspondence: Jan-Willem C. Alffenaar, University of Sydney, Faculty of Medicine and Health, School of Pharmacy, Science Road, Sydney, New South Wales 2006, Australia. E-mail: johannes.alffenaardasydney.edu.au

@ERSpublications

Once weekly bedaquiline, rifapentine and pyrazinamide has sterilising activity and is ready for further exploration in a clinical trial, including appropriate safety and pharmacokinetic assessment https://bit. ly/3ivrY4m

Cite this article as: Alffenaar J-WC, Tiberi S, Cirillo DM, et al. Intermittent regimens for tuberculosis treatment: Back to the Future? Eur Respir J 2020; 56: 2002510 [https://doi.org/10.1183/13993003.025102020].

Despite all efforts to accelerate the response against tuberculosis (TB), many countries are struggling to achieve the milestones of the End TB strategy [1]. The investment required in a strong healthcare system to combat TB is significant. Lack of a short and highly active sterilising TB treatment regimen is the main reason for treatment failure and emergence of drug resistance [2]. With increasing drug resistance, the treatment becomes longer and more difficult to manage due to use of more toxic and less active drugs. Although treatment management is focused on prevention of adverse drug reactions, most patients will experience drug toxicity during treatment [3] which can result in nonadherence.

Optimisation of TB treatment is key to reducing the global burden of TB. Attempts have been made to shorten the current treatment for drug susceptible TB, consisting of isoniazid, rifampicin, pyrazinamide and ethambutol, by adjusting the first line regimen. Replacing ethambutol with moxifloxacin did not result in the foreseen shortening of treatment from 6 months to 4 months [4]; explanations may be low moxifloxacin drug exposure due to rifampicin drug-drug interaction [5] or lack of penetration in caseous lesions [6]. It is expected that shortening of TB treatment is possible in well-defined patients (without cavities and of female gender). Using a combination of moxifloxacin and rifapentine in a 4-month regimen was not non-inferior to the standard first line regimen either [7]. Optimisation of the dosing of the first line drugs has shown favourable results for rifampicin [8], but final results of phase III studies are still pending.

Besides dose optimisation and attempts to replace less-active drugs in regimen with those that are more active, other approaches have been trialled to optimise treatment. Simple regimens requiring less frequent dosing to increase adherence and make direct observation of treatment easier have been studied for the first line regimen. It was shown that thrice-weekly intermittent dosing schedules were associated with increased risk of acquired drug resistance [9], which made the World Health Organization (WHO) decide 
to not recommend these regimens. The acquired resistance could be explained by the fact that suppression of resistance was associated with the rifampicin free peak concentration-to-minimal inhibitory concentration (MIC) ratio exceeding 175 [10]. This can only be achieved at a much higher dosage than traditionally used in first line treatment. The use of a second agent to protect rifampicin is therefore of critical importance. Isoniazid has fulfilled this role as part of the continuation phase of treatment but, despite the combination of rifampicin and isoniazid, acquired resistance still occurs [11]. The complex isoniazid concentration dependent prevention of acquired resistance is partly the underlying problem [12]. As both rifampicin and isoniazid show a considerable variability in drug exposure, personalised dosing could be a solution to overcome the problems experienced with intermittent treatment. However, this would be highly unpractical as tools are not readily available $[13,14]$.

With the recent introduction of new anti-TB drugs like bedaquiline [15], delamanid [16] and pretomanid [17], new highly active drugs have become available to treat TB [18]. Although the new drugs are currently registered for drug-resistant $\mathrm{TB}$, the drugs are trialled for shortening treatment for drug-susceptible TB as well (see trial registered at clinicaltrials.gov as NCT03338621). This would make the step towards the design of a pan-TB treatment regimen easier [19]. Since pharmacokinetics and pharmacodynamics have played a more prominent role in TB drug development, the selection of the combination of drugs and their corresponding dosages is more scientifically based than in the past [20]. This will hopefully prevent the introduction of suboptimal dosages of anti-TB drugs in future regimens. KorT et al. [21] present the findings of their study in which they tested their hypothesis that a once weekly regimen containing bedaquiline, rifapentine and pyrazinamide (figure 1), with or without moxifloxacin or moxifloxacin and clofazimine, has a sterilising activity. The core bactericidal drugs of the regimen, bedaquiline and rifapentine, have both a long half-life and their efficacy is driven by area under the concentration time curve (AUC) to MIC ratio $[22,23]$. The most important finding from their study was that the relapse rate of the experimental once-weekly regimen was significantly lower assessed at 3 months after completion of a 4- or 6-month treatment regimen compared to standard first line treatment [21].

How to translate the results from this preclinical study to clinical practice is an important question. First, as the efficacy of bedaquiline and rifapentine was related to AUC/MIC, the drug exposure in the animal model mimicking the human exposure of the drug needs to be confirmed. In this study the drug-drug interaction between rifapentine and bedaquiline present in humans was mimicked by dose reduction of

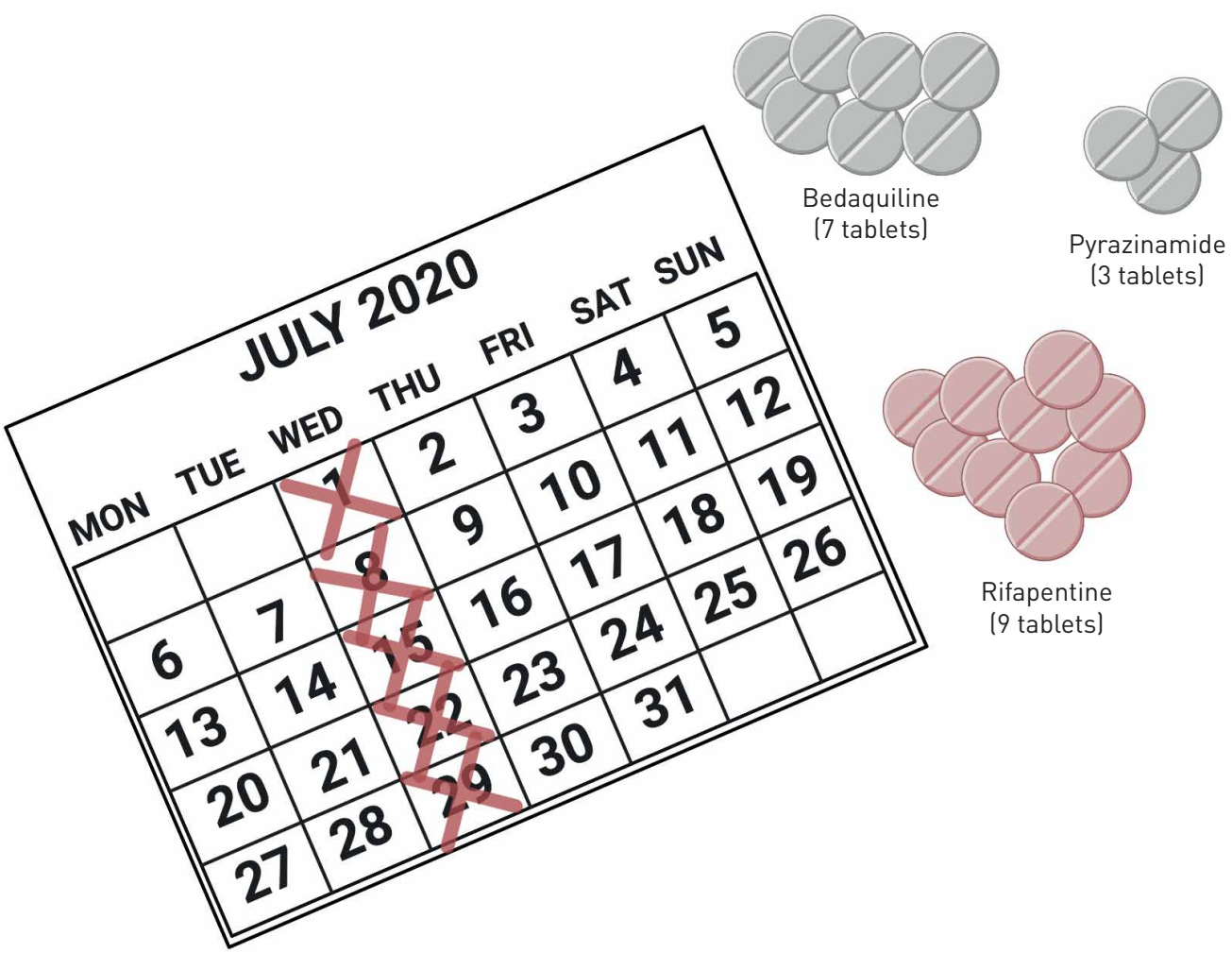

FIGURE 1 Once weekly bedaquiline, rifapentine and pyrazinamide regimen. Artwork by Anne-Grete Märtson. 
bedaquiline. Rifapentine is considered a strong inductor of CYP3A4 [24], while bedaquiline is a substrate [25] resulting in a considerable reduction of AUC of approximately $75 \%$. Although adjusting bedaquiline dose may be considered appropriate, the actual assessment of drug exposure would have been preferred [26]. Second, the preclinical model should resemble the mode of TB disease and relapse rate as seen in humans to be informative for future trial design for the experimental regimen in humans. Use of the appropriate Mycobacterium tuberculosis strain, inoculum and route of infection are equally important to produce relevant results. Use of a control group receiving the standard treatment regimen including isoniazid, rifampicin and pyrazinamide is important for the validity of the results with respect to translation to humans. Third, drug safety will be different in humans than in animals due to underlying clinical condition and co-morbidities and co-administered drugs. When drugs are evaluated in clinical trials, safety of participants is assured by scheduled assessments of critical parameters, as well as a system for reporting of adverse events. In real-life, drug safety assurance is a considerable issue, as resources for assessment are less compared to clinical trials. The introduction of active drug safety monitoring in daily practice has been successfully implemented to evaluate the frequency and severity of adverse events of new drugs like bedaquiline [27]. As KORT et al. [21] rightfully mention, the tested regimen including bedaquiline, clofazimine and moxifloxacin may not be a treatment regimen that fits all patients due to the increased risk of QT prolongation [28]. As more data is collected on both individual drugs and on their combinations, a patient-centred approach will likely be a solution $[29,30]$.

KORT et al. [21] have shown that once weekly bedaquiline, rifapentine and pyrazinamide has sterilising activity and is ready for further exploration in a clinical trial including appropriate safety and pharmacokinetic assessment.

Acknowledgements: The project is part of the activities of the Global Tuberculosis Network (GTN) and of the WHO Collaborating Centre for Tuberculosis and Lung Diseases, Tradate, ITA-80, 2017-2020- GBM/RC/LDA. The authors wish to thank Anne-Grete Märtson for her editorial assistance and for preparing the artwork for figure 1 .

Conflict of interest: None declared.

\section{References}

1 World Health Organization (WHO). Global Tuberculosis Report 2019. Geneva, WHO, 2019.

2 Wallis RS, Maeurer M, Mwaba P, et al. Tuberculosis-advances in development of new drugs, treatment regimens, host-directed therapies, and biomarkers. Lancet Infect Dis 2016; 16: e34-e46.

3 Lan Z, Ahmad N, Baghaei P, et al. Drug-associated adverse events in the treatment of multidrug-resistant tuberculosis: an individual patient data meta-analysis. Lancet Respir Med 2020; 8: 383-394.

4 Gillespie SH, Crook AM, McHugh TD, et al. Four-month moxifloxacin-based regimens for drug-sensitive tuberculosis. N Engl J Med 2014; 371: 1577-1587.

5 Alffenaar J, Gumbo T, Aarnoutse R. Shorter moxifloxacin-based regimens for drug-sensitive tuberculosis. $N$ Engl J Med 2015; 372: 576-577.

6 Prideaux B, Via LE, Zimmerman MD, et al. The association between sterilizing activity and drug distribution into tuberculosis lesions. Nat Med 2015; 21: 1223-1227.

7 Jindani A, Harrison TS, Nunn AJ, et al. High-dose rifapentine with moxifloxacin for pulmonary tuberculosis. N Engl J Med 2014; 371: 1599-1608.

8 Svensson EM, Svensson RJ, Te Brake LHM, et al. The potential for treatment shortening with higher rifampicin doses: Relating drug exposure to treatment response in patients with pulmonary tuberculosis. Clin Infect Dis 2018; 67: 34-41.

9 Menzies D, Benedetti A, Paydar A, et al. Effect of duration and intermittency of rifampin on tuberculosis treatment outcomes: a systematic review and meta-analysis. PLoS Med 2009; 6: e1000146.

10 Gumbo T, Louie A, Deziel MR, et al. Concentration-dependent Mycobacterium tuberculosis killing and prevention of resistance by rifampin. Antimicrob Agents Chemother 2007; 51: 3781-3788.

11 Pasipanodya JG, McIlleron H, Burger A, et al. Serum drug concentrations predictive of pulmonary tuberculosis outcomes. J Infect Dis 2013; 208: 1464-1473.

12 Gumbo T, Louie A, Liu W, et al. Isoniazid bactericidal activity and resistance emergence: integrating pharmacodynamics and pharmacogenomics to predict efficacy in different ethnic populations. Antimicrob Agents Chemother 2007; 51: 2329-2336.

13 Ghimire S, Bolhuis MS, Sturkenboom MGG, et al. Incorporating therapeutic drug monitoring into the World Health Organization hierarchy of tuberculosis diagnostics. Eur Respir J 2016; 47: 1867-1869.

14 Alffenaar J-WC, Gumbo T, Dooley KE, et al. Integrating pharmacokinetics and pharmacodynamics in operational research to end tuberculosis. Clin Infect Dis 2019; 70: 1774-1780.

15 Borisov SE, Dheda K, Enwerem M, et al. Effectiveness and safety of bedaquiline-containing regimens in the treatment of MDR- and XDR-TB: a multicentre study. Eur Respir J 2017; 49: 1700387.

16 Skripconoka V, Danilovits M, Pehme L, et al. Delamanid improves outcomes and reduces mortality in multidrug-resistant tuberculosis. Eur Respir J 2013; 41: 1393-1400.

17 Dawson R, Diacon AH, Everitt D, et al. Efficiency and safety of the combination of moxifloxacin, pretomanid (PA-824), and pyrazinamide during the first 8 weeks of antituberculosis treatment: a phase $2 \mathrm{~b}$, open-label, partly randomised trial in patients with drug-susceptible or drug-resistant pulmonary tuberculosis. Lancet 2015; 385: 1738-1747.

18 Caminero JA, García-Basteiro AL, Rendon A, et al. The future of drug-resistant tuberculosis treatment: learning from the past and the 2019 World Health Organization consolidated guidelines. Eur Respir J 2019; 54: 1901272. 
19 Lienhardt C, Nahid P, Rich ML, et al. Target regimen profiles for treatment of tuberculosis: a WHO document. Eur Respir J 2017; 49: 1602352.

20 Van Ingen J, Aarnoutse RE, Donald PR, et al. Why do we use $600 \mathrm{mg}$ of rifampicin in tuberculosis treatment? Clin Infect Dis 2011; 52: e194-e199.

21 Kort F, Fournier Le Ray L, Chauffour A, et al. Fully weekly antituberculosis regimen: a proof-of-concept study. Eur Respir J 2020; 56: 1902502.

22 Savic RM, Weiner M, MacKenzie WR, et al. Defining the optimal dose of rifapentine for pulmonary tuberculosis: exposure-response relations from two phase II clinical trials. Clin Pharmacol Ther 2017; 102: 321-331.

23 Rouan MC, Lounis N, Gevers T, et al. Pharmacokinetics and pharmacodynamics of TMC207 and its N-desmethyl metabolite in a murine model of tuberculosis. Antimicrob Agents Chemother 2012; 56: 1444-1451.

24 Svensson E, Murray S, Karlsson M, et al. Rifampicin and rifapentine significantly reduce concentrations of bedaquiline, a new anti-TB drug. J Antimicrob Chemother 2015; 70: 1106-1114.

25 van Heeswijk RPG, Dannemann B, Hoetelmans RMW. Bedaquiline: a review of human pharmacokinetics and drug-drug interactions. J Antimicrob Chemother 2014; 69: 2310-2318.

26 Alffenaar JWC, Akkerman OW, Tiberi S, et al. Should we worry about bedaquiline exposure in the treatment of multidrug-resistant and extensively drug-resistant tuberculosis? Eur Respir J 2020; 55: 1901908.

27 Borisov S, Danila E, Maryandyshev A, et al. Surveillance of adverse events in the treatment of drug-resistant tuberculosis: first global report. Eur Respir J 2019; 54: 1901522.

28 Yoon HY, Jo KW, Nam GB, et al. Clinical significance of QT-prolonging drug use in patients with MDR-TB or NTM disease. Int J Tuberc Lung Dis 2017; 21: 996-1001.

29 Pontali E, Sotgiu G, Tiberi S, et al. Cardiac safety of bedaquiline: a systematic and critical analysis of the evidence. Eur Respir J 2017; 50: 1701462.

30 Zweijpfenning SMH, Van Groningen H, Van Ingen J, et al. Clofazimine does not lead to significant QT interval prolongation: A multicentre study. Eur Respir J 2018; 52: 1801386. 